\title{
Conformational Molecular Switch of the Azobenzene Molecule: A Scanning Tunneling Microscopy Study
}

\author{
Byoung-Young Choi, ${ }^{1}$ Se-Jong Kahng, ${ }^{2}$ Seungchul Kim, ${ }^{3}$ Hajin Kim, ${ }^{1}$ Hyo Won Kim, ${ }^{1}$ \\ Young Jae Song, ${ }^{1}$ Jisoon Ihm, ${ }^{3}$ and Young Kuk ${ }^{1, *}$ \\ ${ }^{1}$ Department of Physics and Center for Science in Nanometer Scale, Seoul National University, Seoul 151-747, Korea \\ ${ }^{2}$ Department of Physics, Korea University, Seoul, 136-701, Korea \\ ${ }^{3}$ Department of Physics, Seoul National University, Seoul, 151-747, Korea
}

(Received 27 October 2005; published 21 April 2006)

\begin{abstract}
We propose to utilize azobenzene as a nanomolecular switch which can be triggered by transmitting electrons above threshold biases. The effect is explained by an electron impact trans-cis conformational change of the isolated azobenzene molecules. The molecular electronic states of both isomers have been measured with spatially resolved scanning tunneling microscopy or spectroscopy, leading to suggested transition pathways of the electron-induced isomerization.
\end{abstract}

Despite remarkable progress in silicon nanoprocessing technology, many believe molecular electronics will surface as an alternative device technology. Many groups have tried to build hybrid-molecular and monomolecular devices such as transistors, rectifiers and switches by applying existing patterning techniques or using molecular selfassembly [1]. Conjugated molecular chains exhibit good conductivity with $\pi$ bonding for the carrier transport [2]. If two disconnected parts of a conducting molecular chain are connected by a bond with two coupled potential energy surfaces with 2 degrees of freedom, the resulting chain will reveal nonlinear characteristics $[3,4]$. It has been shown experimentally in a scanning tunneling microscopy (STM) that these kinds of molecules switch between two potential energy minima and function as nanomolecular switches [5-7]. It has been suggested that excitation by an applied electric field [8] or a laser field [3] is the trigger for the molecular switch and resultant conformational change (CC) [Fig. 1(a)]. Chemical isomers are the most appropriate target molecules, because they have the same molecular formula and connectivity but different geometric structures and conductivities.

Azobenzene (AB) was chosen to study the CC. It has two isomers which are determined by dihedral angles between two phenyl rings and the $\mathrm{CN}=\mathrm{NC}$ plane $[9,10]$. Trans-azobenzene (tAB) can be transformed to cis-azobenzene (cAB) by rotating or inverting a phenyl ring through the excitation to the $\pi-\pi^{*}\left(S_{2}\right)$ state with ultraviolet photons or the $n-\pi^{*}\left(S_{1}\right)$ state with green photons [11,12]. A reverse isomerization from $\mathrm{CAB}$ to $\mathrm{tAB}$ was reported to have been achieved either by visible light or thermal excitation [13]. In this Letter, we propose that the reversible conformational change in $\mathrm{AB}$ can be utilized as a nanomolecular switch [Fig. 1(b)]. The switch can be triggered by tunneling electrons with a specific bias in the STM geometry. The electronic structures of two potential energy minima were measured using spatially resolved scanning tunneling spectroscopy (SR-STS), and explained with our first-principles study. Suggestions for the pathways of the isomerization are based on the experimental data and theoretical calculations.

The experimental details have been described elsewhere [14]. Briefly, we leaked gas phase $\mathrm{tAB}$ on an atomically clean $\mathrm{Au}(111)$ surface at $100 \mathrm{~K}$ and then performed STM and position-resolved spectroscopy at $5 \mathrm{~K}$ in an ultrahigh vacuum $\left(<1 \times 10^{-10}\right.$ Torr $)$. In order to understand the observed structures and spectra in more detail, we performed a first-principles pseudopotential electronic structure calculation based on density functional theory [15] with a local density approximation [16]. We relaxed the atomic positions using the total energy minimization method [17] and expanded the wave functions in a double-zeta plus polarization basis set with an energy cutoff (for real space mesh points) of $80 \mathrm{Ry}$.

It was recently reported that $\mathrm{tAB}$ has been grown on an $\mathrm{Au}(111)$ surface with various intermolecular assemblies (a)

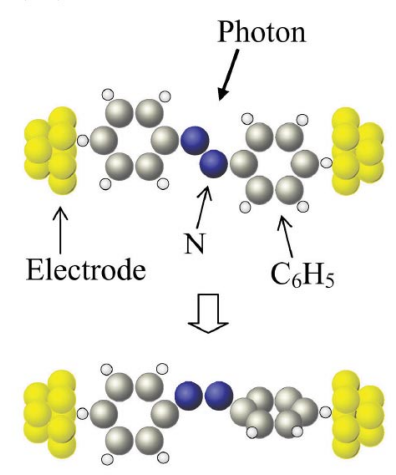

(b)

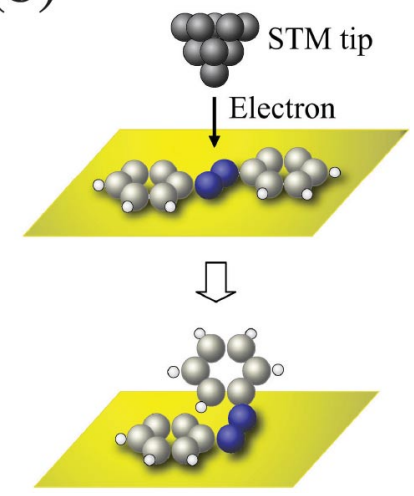

FIG. 1 (color online). (a) A schematic of a light-driven molecular switch based on the conformational change of an azobenzene $(\mathrm{AB})$ molecule in contact with two metallic electrodes. (b) A concept of electron-induced isomerization of an $\mathrm{AB}$ molecule adsorbed onto a metallic surface with a scanning tunneling microscope. 
such as a chain phases monolayer [18]. In the present work, we focused on an intramolecular conformational change of isolated AAB. The STM images in Fig. 2(a) and the left side inset of Fig. 2(b) show isolated tAB molecules adsorbed onto the herringbone edges of an $\mathrm{Au}(111)$ surface, which look like dumbbells as reported earlier [18]. The lobes of the dumbbell are attributed to the phenyl rings of tAB. The nitrogen-nitrogen (NN) double bond constitutes a node structure in the STM image because of weak local electron density of states (LDOS) above the Fermi level. Figure 2(b) shows that we were able to change the shape of $\mathrm{tAB}$ with a bias voltage of lower than $\sim-1.5 \mathrm{~V}$, i.e., an energetic electron beam. By positioning the STM tip on top of a AAB center, we were able to measure the tunneling current as we swept the sample bias. As expected, the measured tunneling current increased monotonically at a sample bias of up to $2 \mathrm{~V}$. At negative bias, we observed an abrupt jump of the tunneling current at $\sim-1.5 \mathrm{~V}$, as shown in Fig. 2. After this abrupt increase, a new molecule appeared instead of tAB (Fig. 2 inset). The measured jumping rate was order of

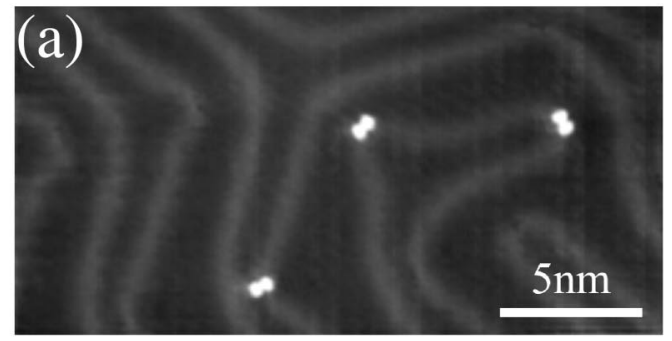

(b)

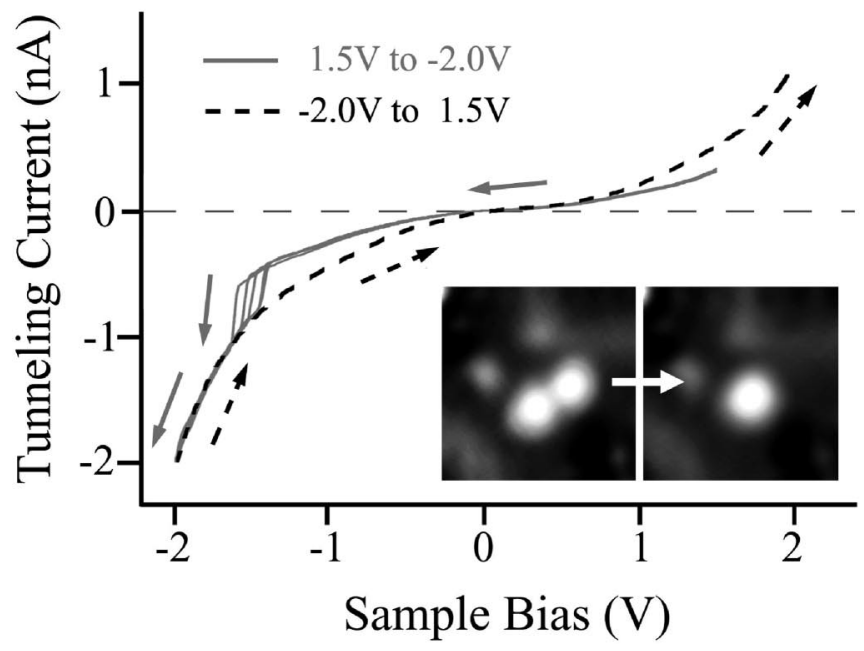

FIG. 2. (a) A large scale image $\left(V_{s}=1 \mathrm{~V}, I_{t}=50 \mathrm{pA}\right)$ of 3 trans-azobenzenes on $\mathrm{Au}(111)$. (b) A current-voltage $(I-V)$ curve obtained at the center of tAB (left inset) and a newly produced molecule (right inset) by sweeping the voltage twice; from 1.5 to $-2.0 \mathrm{~V}$ on $\mathrm{ABB}$ (solid line) and from -2.0 to $2.0 \mathrm{~V}$ on the new product (dashed line). Insets: Topographic images $(2.2 \mathrm{~nm} \times 2.2 \mathrm{~nm})$ before the $I-V$ measurement (left) and after a current jump (right), taken at $V_{s}=1 \mathrm{~V}$ and $I_{t}=70 \mathrm{pA}$.
$10^{-18}$ per electron, resulting in a cross section of $\sim 10^{-24} \mathrm{~cm}^{2}$. This new molecule was reverted to tAB with the positive sample bias above $2.5 \mathrm{~V}$ and a similar tunneling current versus bias voltage relation was obtained.

We determined that the new image that appeared was due to (1) a change in the STM tip, (2) fragments of tAB, or (3) the presence of $\mathrm{cAB}$. Case (1) was ruled out since the surroundings of the molecules were not changed after the current jump. Case (2) was also excluded because this process is reversible, as shown in Fig. 3(a). We repeated the tunneling electron-induced transformation and reverse transition in sequence with voltage pulses of $\sim 100 \mathrm{~ms}$ [Fig. 3(a)]. Figure 3(b) shows the corresponding height cross section of two molecules along their central axes and the calculated adsorption geometries are shown in Fig. 3(c). The calculations indicate that $\mathrm{tAB}$ lies parallel to the surface as shown in Fig. 3(c). We tested 8 different positions of $\mathrm{tAB}$ with respect to the gold surface. In the lowest energy configuration, three carbon atoms in each phenyl ring are located almost on top of gold atoms and strongly hybridized with them, and the binding energy of $\mathrm{tAB}$ to the gold surface is $2.77 \mathrm{eV}$. In $\mathrm{cAB}, 11$ different configurations including the cases of no phenyl ring being in parallel with the surface were tested. In the lowest energy, one phenyl ring runs parallel to the substrate while the other ring folds up at an angle of $\sim 111^{\circ}$ and the binding energy of cAB to the gold surface is $1.94 \mathrm{eV}$, as shown in Fig. 3(c). Since such a folded (nonplanar) configuration of $\mathrm{cAB}$ was in good agreement with the asymmetric oval shape and height profile in Fig. 3(b), we regarded the new product as a $\mathrm{CAB}$ molecule. We note that another relaxed configuration whose binding energy was comparable to that of the folded configuration above had both phenyl rings lying parallel with the surface [19], but its STM topographic image did not match our experimental data [20]. Figure 3(d) shows a spatially controlled isomerization on a selected $\mathrm{ABB}(t 2)$ and indicates again that the STM tip was unchanged. More decisive evidence is presented below.

In order to confirm the isomerization process, we performed an SR-STS measurement to map the electronic structures of tAB and cAB. Figs. 4(a) and 4(b) show 128 $d I / d V$ spectra stacked side by side, which were obtained along the central axes of $\mathrm{tAB}$ and $\mathrm{cAB}$. In SR-STS, the charge distribution of a molecular state can be examined with spatial resolution. For example, LDOS contributed by the two phenyl rings of tAB is separated spatially at $1.5 \mathrm{eV}$. In Figs. 4(c) and 4(d), the measured $(d I / d V) /(I / V)$ data (a normalized $d I / d V$ ) for $\mathrm{tAB}$ and $\mathrm{cAB}$ at the centers of the molecules are presented in comparison with theoretical LDOS curves. The calculated highest occupied molecular orbital (HOMO) level in tAB is broadened by hybridization with an $\mathrm{Au}(111)$ state and gives rise to peak structures near $-1.2 \mathrm{eV}$, in good agreement with experiment. The calculated peaks at lowest unoccupied molecular orbital level is smaller than the measured one and contributes to peaks between -0.1 and $0.9 \mathrm{eV}$. A small peak around zero 
(a)

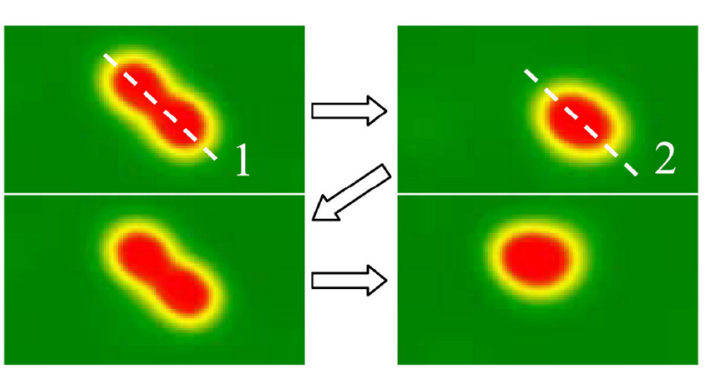

(b)

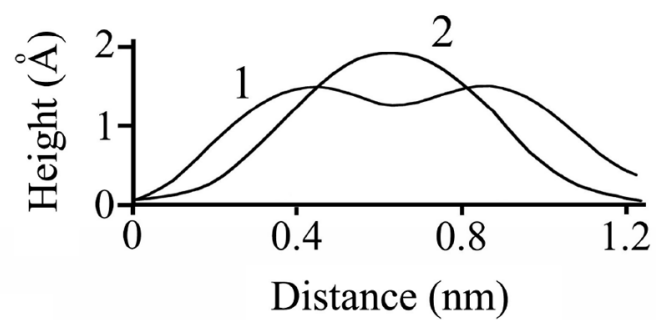

(c)

Top View
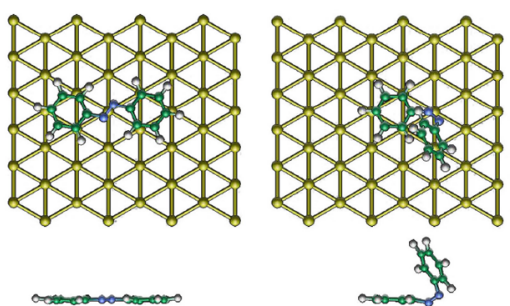

Side View

trans

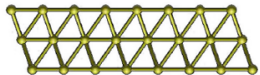

cis

(d)

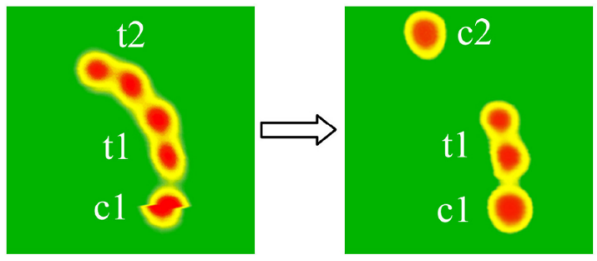

FIG. 3 (color online). (a) STM images $(2.4 \mathrm{~nm} \times 1.3 \mathrm{~nm})$ of $\mathrm{tAB}$ and an electron-induced product, attributed to $\mathrm{cAB}$. The voltage pulse was $-2 \mathrm{~V}$ (upper left to upper right and lower left to lower right) and $2.5 \mathrm{~V}$ (upper right to lower left). (b) The cross sections of the molecules in (a) along the dashed arrows. "1" represents the upper left molecule and " 2 " the upper right. (c) The proposed adsorption geometries of $\mathrm{tAB}$ and $\mathrm{cAB}$ on $\mathrm{Au}(111)$. (d) A spatially controlled isomerization on a selected tAB. " $t 2$ " ( $\mathrm{tAB}$ ) was transformed to " $c 2$ " ( $\mathrm{cAB}$ ) by electron beam with voltage of $-2 \mathrm{~V}$, while other molecules were unchanged. " $c 1$ " is an existing $\mathrm{cAB}$.

bias can be found in experimental $d I / d V$, which mainly consists of an STM tip state frequently shown when a tungsten tip is used.

Unlike tAB, cAB on Au(111) in Fig. 4(d) clearly shows that the measured HOMO peak is shifted upward by $\sim 0.5 \mathrm{eV}$ with respect to the theoretical LDOS [a shifted curve is shown in Fig. 4(d)]. A clear explanation for this shift is not available yet. There are usually two origins of the shift of $d I / d V$ : (1) For a given external bias, a partial potential drop may occur between the molecule (cAB) and its substrate, or (2) a charge transfer may take place at the interfaces. Case (1) is excluded here because the observed upward shift at a negative sample bias is opposite to the expected downward shift for a potential drop. A possible scenario for explaining the shift is an electron transfer from the substrate to $\mathrm{CAB}$. This is consistent with the fact that no states are observed at $V=0$ and the cAB acts as a leaky capacitor capable of storing extra charges for a certain time maintaining a nonequilibrium situation. The observed upshift of $0.5 \mathrm{eV}$ corresponds to a one electron $(1.6 \times$ $10^{-19} \mathrm{C}$ ) transfer to $\mathrm{cAB}$, assuming the capacitance of the system to be $3 \times 10^{-19} \mathrm{~F}$. This observation suggests that $\mathrm{CAB}$ might be behaving as a quantum dot in the STM setting with nonresonant tunneling electrons.

Understanding the detailed mechanism of the isomerization is a challenging task which we have tried to undertake with STM. There are, however, still controversial aspects of our results. First, the polarity dependence was previously reported in the conformational transformation of a dye molecule [21]. Second, the bias voltage used for the electron-induced isomerization in the present work is lower than the photon energy $(2.8-3.9 \mathrm{eV})$ required for photoisomerization $[11,12,22]$. And lastly, the estimated cross section of electron-induced isomerization was also (a)

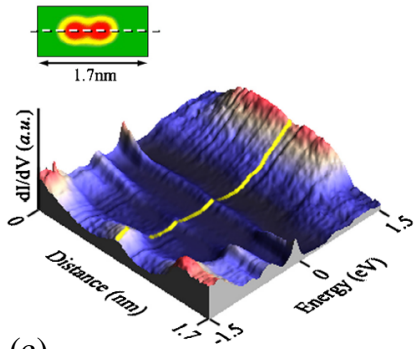

(c)

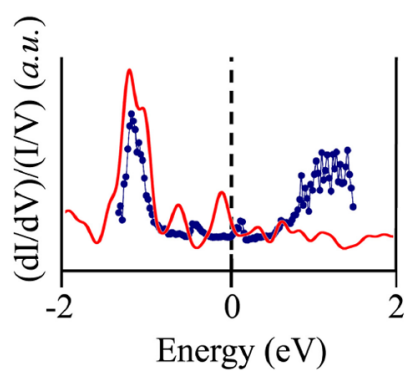

(b)

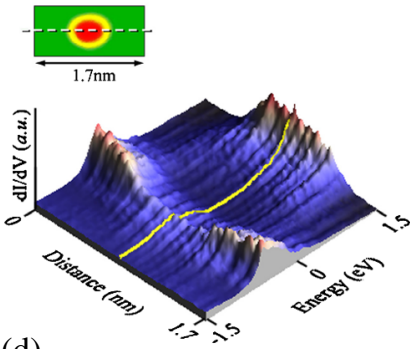

(d)

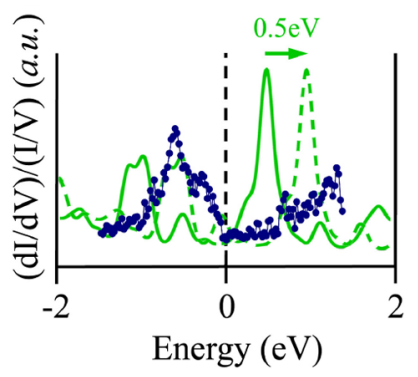

FIG. 4 (color online). (a), (b) Three dimensional SR-STS maps along the central axes of $\mathrm{tAB}$ and $\mathrm{cAB}$, respectively, and their topographic images (insets, $1.7 \mathrm{~nm} \times 0.8 \mathrm{~nm}$ ). The $z$ axis is the measured $d I / d V$, the $x$ axis is the scanned length of $1.7 \mathrm{~nm}$, and the $y$ axis is the scanned energy of $-1.5-1.5 \mathrm{eV}$. (c), (d) The experimental (solid circle) and theoretical (solid line) $(d I / d V) /(I / V)$ spectra of $\mathrm{tAB}$ and $\mathrm{cAB}$ at the center of the molecules. A dashed line in (d) is the theoretical LDOS spectrum shifted by $+0.5 \mathrm{~V}$. 
smaller than that of photoisomerization $\left(\sim 10^{-18} \mathrm{~cm}^{2}\right)$ [23]. There are several scenarios to explain the difference of electron-induced isomerization. (1) It was reported that isomerization characteristics such as the transition pathway and the yield depend on the type and viscosity of solvent and the pressure of mixed gases [11,24]. By this inference from this information, we may surmise that metallic surface or local substrate geometry might be causing reformation of the molecular state, resulting in reduction of the resonant excitation barrier for the isomerization. (2) The isomerization may take place by crossing over the potential barrier between trans and cis states without resonant excitation to the $S_{1}$ or $S_{2}$ state. The excitation energy, required in this case, was reported to be only $\sim 1.6 \mathrm{eV}$ [25]. (3) It was suggested that, theoretically, vibrational excitation may trigger the isomerization [26]. Isomerization caused by inelastic electron tunneling might provide a new mechanism for the process. The validity of these scenarios can be studied further by performing experiments on patterned or other metallic surfaces in the future.

In summary, we showed that azobenzene is a good candidate for use as a molecular switch. This switch can even be triggered by a local tunneling electron, so it is a true nano switch. The $\mathrm{tAB}$ state was transformed to the $\mathrm{cAB}$ state reversibly either by electron impact resonant excitation or by vibrational excitation, different from photoisomerization.

The authors thank Michael F. Crommie at University of California, Berkeley for helpful discussion and Karina Morgenstern at the University of Hanover for a copy of her paper prior to publication. This work was supported by the Creative Research Initiatives Program of Korean Ministry of Science and Technology through Korean Science and Engineering Foundation, the SRC program (CNNC of Sungkyunkwan University), and the National R\&D Project for Nano-Science and Technology.

*Corresponding author.

Email address: ykuk@phya.snu.ac.kr

[1] C. Joachim, J.K. Gimzewski, and A. Aviram, Nature (London) 408, 541 (2000); J. R. Heath and M. A. Ratner, Phys. Today 56, No. 5, 43 (2003).

[2] C. K. Chiang, C. R. Fincher, Jr., Y. W. Park, A. J. Heeger, H. Shirakawa, E. J. Louis, S.C. Gau, and A. G. MacDiarmid, Phys. Rev. Lett. 39, 1098 (1977).

[3] F. Grossmann, L. Feng, G. Schmidt, T. Kunert, and R. Schmidt, Europhys. Lett. 60, 201 (2002).

[4] A. Troisi and M. A. Ratner, Nano Lett. 4, 591 (2004).
[5] Z. J. Donhauser, B. A. Mantooth, K. F. Kelly, L. A. Bumm, J. D. Monnell, J. J. Stapleton, D. W. Price, Jr., A. M. Rawlett, D. L. Allara, J.M. Tour, and P.S. Weiss, Science 292, 2303 (2001).

[6] G. K. Ramachandran, T. J. Hopson, A. M. Rawlett, L. A. Nagahara, A. Primak, and S. M. Lindsay, Science 300, 1413 (2003).

[7] M. Lastapis, M. Martin, D. Riedel, L. Hellner, G. Comtet, and G. Dujardin, Science 308, 1000 (2005).

[8] B. I. Schneider, T. N. Rescigno, and C. W. McCurdy, Phys. Rev. A 42, 3132 (1990).

[9] T. Nägele, R. Hoche, W. Zinth, and J. Wachtweitl, Chem. Phys. Lett. 272, 489 (1997).

[10] S. Monti, G. Orlandi, and P. Palmieri, Chem. Phys. 71, 87 (1982).

[11] C.-W. Chang, Y.-C. Lu, T.-T Wang, and E. W.-G. Diau, J. Am. Chem. Soc. 126, 10109 (2004).

[12] Y. Hirose, H. Yui, and T. Sawada, J. Phys. Chem. A 106, 3067 (2002).

[13] T. Ikegami, N. Kurita, H. Sekino, and Y. Ishikawa, J. Phys. Chem. A 107, 4555 (2003).

[14] J. Lee, H. J. Kim, G. Kim, Y.-W. Son, J. Ihm, S. J. Kahng, H. Kato, Z. W. Wang, T. Okazaki, H. Shinohara, and Y. Kuk, Nature (London) 415, 1005 (2002).

[15] W. Kohn and L. J. Sham, Phys. Rev. 140, A1133 (1965).

[16] D. M. Ceperley and B. J. Alder, Phys. Rev. Lett. 45, 566 (1980).

[17] N. Troullier and J.L. Martins, Phys. Rev. B 43, 1993 (1991).

[18] A. Kirakosian, M.J. Comstock, J. Cho, and M.F. Crommie, Phys. Rev. B 71, 113409 (2005).

[19] Jörg Henzl, Michael Mehlhorn, Heiko Gawronski, KarlHeinz Rieder, and Karina Morgenstern, Angew. Chem., Int. Ed. 45, 603 (2006).

[20] It is interesting to note that, in the case of an azobenzene derivative with $\mathrm{NO}_{2}$ and $\mathrm{NH}_{2}$ group (called Disperse Orange 3) in Ref. [19], the cis structure was shown to have both phenyl rings lying parallel with the surface. Our calculation for cis-Disperse Orange 3 indicated that oxygen atoms in the $\mathrm{NO}_{2}$ group, by forming strong bonds with substrate atoms, stabilized a planar structure in agreement with Ref. [19].

[21] X. H. Qiu, G. V. Nazin, and W. Ho, Phys. Rev. Lett. 93, 196806 (2004).

[22] C. Zhang, M.H. Du, H.P. Cheng, X.G. Zhang, A.E. Roitberg, and J. L. Krause, Phys. Rev. Lett. 92, 158301 (2004).

[23] T. G. Pedersen, P. M. Johansen, and H. C. Pedersen, J. Opt. A Pure Appl. Opt. 2, 272 (2000).

[24] A. Meyer, J. Schroeder, and J. Troe, J. Phys. Chem. A 103, 10528 (1999).

[25] E. R. Talaty and J. C. Fargo, Chem. Commun. (London) 2, 65 (1967).

[26] S. Tanaka, S. Itoh, and N. Kurita, Chem. Phys. Lett. 362, 467 (2002). 